\title{
8
}
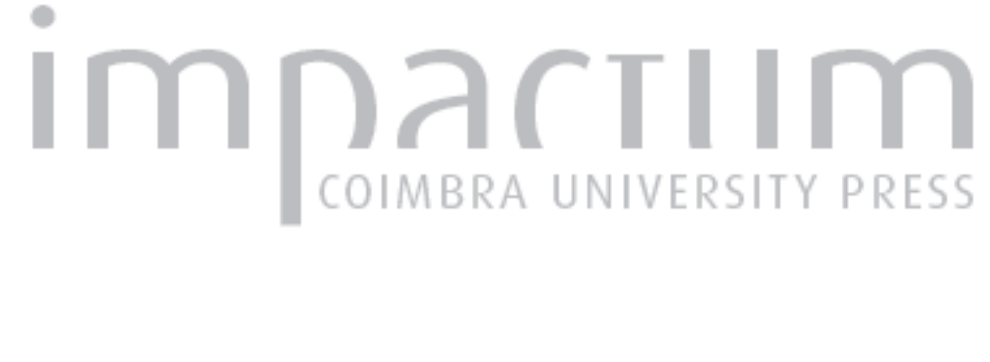

\section{Lesión vertebromedular cervical grave tras colisión a baja velocidad}
Autor(es): $\quad$ Hernando Lorenzo, A; García-Nieto Gómez-Guillamón, F; Wilhelmi Ayza, $\mathrm{M}$; Menchaca Anduaga, A

Publicado por: Imprensa da Universidade de Coimbra

URL

persistente:

URI:http://hdl.handle.net/10316.2/42157

DOI:

DOI:http://dx.doi.org/10.14195/1647-8630_26_1

Accessed : $\quad$ 26-Apr-2023 15:23:03

A navegação consulta e descarregamento dos títulos inseridos nas Bibliotecas Digitais UC Digitalis, UC Pombalina e UC Impactum, pressupõem a aceitação plena e sem reservas dos Termos e Condições de Uso destas Bibliotecas Digitais, disponíveis em https://digitalis.uc.pt/pt-pt/termos.

Conforme exposto nos referidos Termos e Condições de Uso, o descarregamento de títulos de acesso restrito requer uma licença válida de autorização devendo o utilizador aceder ao(s) documento(s) a partir de um endereço de IP da instituição detentora da supramencionada licença.

Ao utilizador é apenas permitido o descarregamento para uso pessoal, pelo que o emprego do(s) título(s) descarregado(s) para outro fim, designadamente comercial, carece de autorização do respetivo autor ou editor da obra.

Na medida em que todas as obras da UC Digitalis se encontram protegidas pelo Código do Direito de Autor e Direitos Conexos e demais legislação aplicável, toda a cópia, parcial ou total, deste documento, nos casos em que é legalmente admitida, deverá conter ou fazer-se acompanhar por este aviso.

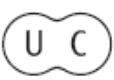




\title{
Lesión vertebromedular cervical grave tras colisión a baja velocidad
}

\author{
A Hernando Lorenzoํㅜ F García-Nieto Gómez-Guillamón², \\ M Wilhelmi Ayza ${ }^{3}$, A Menchaca Anduaga ${ }^{4}$
}

Resumo: Lesão vértebro medular cervical grave após colisão a baixa velocidade.

0 estado anterior de uma vítima (por exemplo o caso de espondilite anquilosante aqui apresentado), pode condicionar de forma muito importante a evolução de um traumatismo relativamente leve, como uma colisão posterior a baixa velocidade, produzindo consequências de uma gravidade desproporcionada, o que deve ser considerado do ponto de vista médico-legal ao estabelecer o nexo de causalidade.

Palavras-chave: Fratura cervical baixa; colisão traseira a baixa velocidade; espondilite anquilosante; nexo causal.

Summary: Severe cervical spinal cord injury after low speed collision.

The previous health state (i.e. the case of ankylosing spondylitis here presented), can modify very much the outcome after a relatively mild trauma, as a low speed rear-end collision, with an out of proportion severity, what should be considered in the medico-legal analysis to establish the causal relationship.

Key-words: Lower cervical fracture; low speed rear-end collision; ankylosing spondylitis; causal relationship.

1 Médico. Especialista en Medicina Intensiva y Cardiología. Magister Universitario en Valoración del Daño Corporal y en Medicina de los Seguros Privados - Madrid

2 Médico. Especialista en Medicina Intensiva y en Anestesiología y Reanimación. Magister Universitario en Valoración del Daño Corporal y en Medicina de los Seguros Privados - Madrid

3 Médico. Especialista en CirugíaCardíovascular. Magister Universitario en Valoración del Daño Corporal Madrid

4 Enfermera.Directora de Enfermería del SUMMA 111 (Sistema de Emergencia Médica de la Comunidad de Madrid). 


\section{INTRODUCCION}

Se presenta un caso de fractura vertebral cervical baja en un conductor, tras colisión por alcance a baja velocidad en paciente con espondilitis anquilosante; se revisa la literatura, destacando la importancia del "estado anterior", desde el punto devista asistencial, para sospechar posibles lesiones, así como para establecer la relación de nexo causal, desde el punto de vista pericial y médico-legal.

\section{DESCRIPCION DEL CASO}

Se produjo un accidente de circulación consistente en la colisión por alcance posterior de un turismo Opel Astra de menos de un año de antigüedad, que presentaba como daños una pequeña abolladura en la defensa delantera que afectaba a matrícula y a parrillabaja y que colisionó por alcance posterior a un vehículo Seat Ibiza 1.4 de 15 años de antigüedad, el cual presentaba como daños pequeña abolladura que afecta a la defensa trasera y a la aleta trasera derecha.

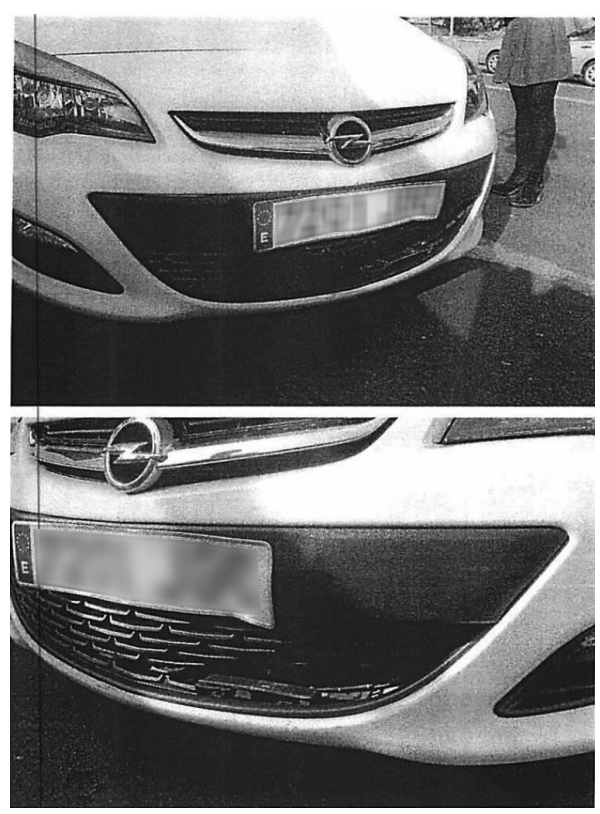

Fig. 1 - Opel Astra 
$11 \mid \begin{aligned} & \text { Lesión vertebromedular cervical grave } \\ & \text { tras colisión a baja velocidad }\end{aligned}$
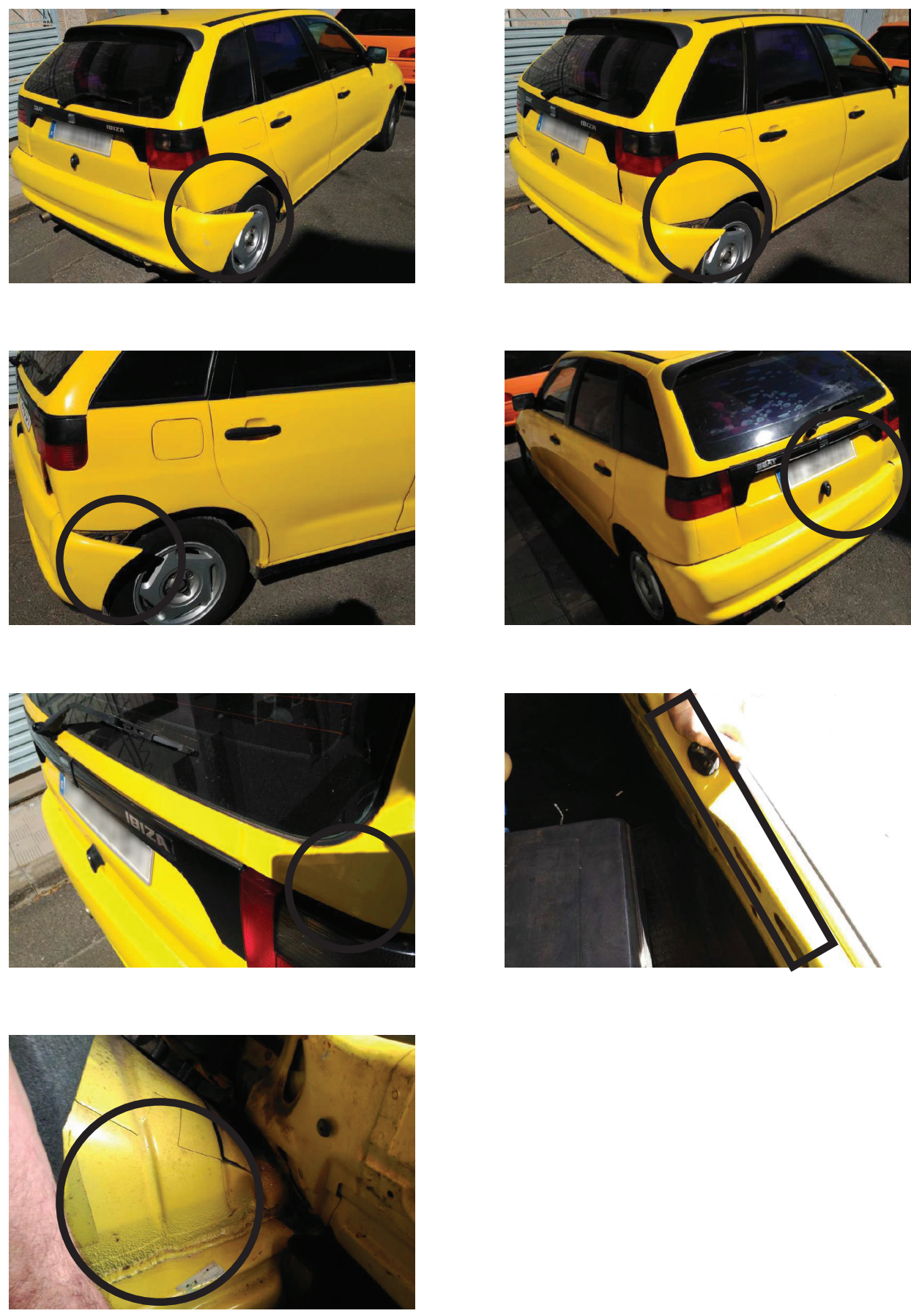

Fig. 2 - Seat Ibiza 
El accidente se produjo cuando estando parado el turismo Seat Ibiza de color amarillo, en el semáforo, el cual estaba en rojo, fue golpeado por el turismo Opel Astra.

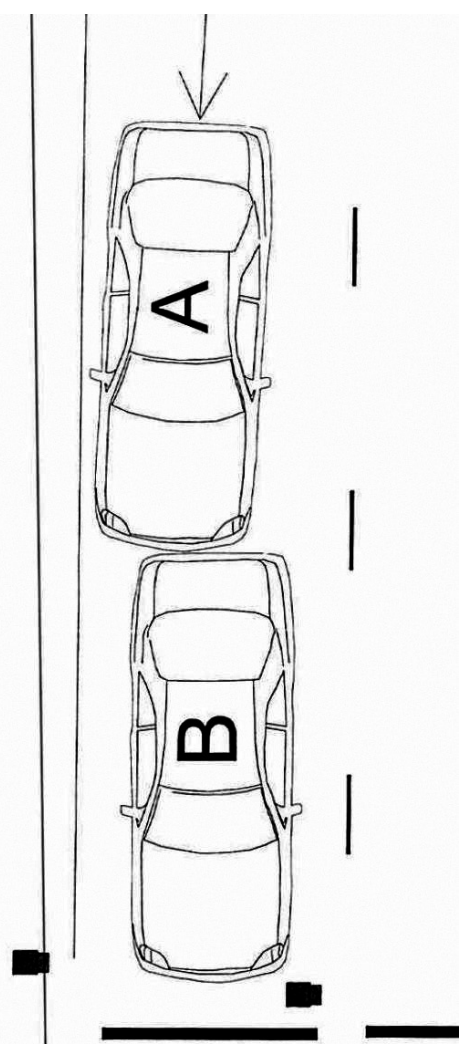

Fig. 3 - El accidente

Como consecuencia del accidente, aparte de los daños en los vehículos, un varón de 56 años de edad, conductor del turismo Seat Ibiza,acudió a Urgencias Hospitalarias; se refiere que llegó a Urgencias sin ningún tipo de déficit neurológico, sin déficit de vías sensitivo motoras largas ni piralidalismo, presentando a la exploración complementaria fractura bilateral de elementos posteriores a nivel de articulación interapofisaria C6-C7, separación severa de cuerpos vertebrales $\mathrm{C6}$-C7 con imagen de "bostezo" y angulación de columna cervical a dicho nivel (fractura tipo B3 - clasificación de la AO) 


\section{Anterior tension band injury}
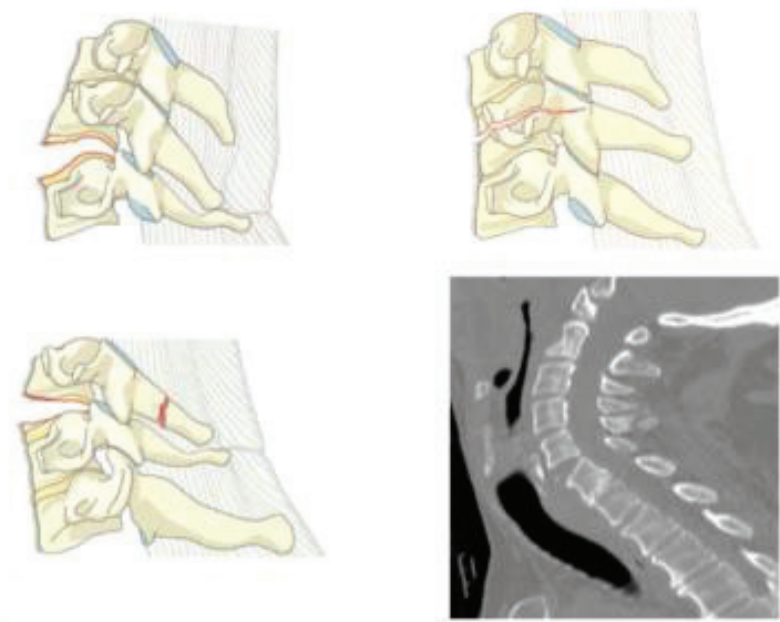

NOTA: lesión similar, pero que NO corresponde al lesionado que comentamos

Fig. 4 - Distraction injuries

Fue intervenido quirúrgicamente, realizándo se laminectomía de C5-C6, hemilaminectomía de $\mathrm{C}$, implante de tornillo sen masas laterales desde $\mathrm{C} 4$ a C7, y por vía anterior colocación de placa en C5-C6 y C7-D1.

El paciente durante la intervención presentó caída de potenciales y tras la intervención quirúrgica se observó tetraparesia que actualmente está en fase de recuperación.

El paciente presentaba entre sus antecedentes espondilitis anquilosante, habiendo sido intervenido quirúrgicamente de fractura de tibia y peroné.

La espondilitis anquilosante (EA) es una forma de artritis que afecta las articulaciones de la columna vertebral. Su nombre viene de las palabras griegas ankylos, que significa rigidez de una articulación y spondylo, que significa vértebra. La espondilitis causa inflamación (enrojecimiento, calor, hinchazón y dolor) en la columna vertebral o en las vértebras.

Afecta principalmente al esqueleto axial (articulaciones sacroiliacas y columna vertebral) y es una enfermedad inflamatoria crónica duradera a lo largo de la vida, que a menudo comienza en adultos jóvenes, siendo la edad de comienzo menos de 30 años en el $80 \%$ de pacientes. 
Así, la espondilitis anquilosante (EA), típicamente afecta a gente jóven, comenzando entre los 15 y 30 años; la edad promedio de comienzo de la enfermedad es 24 años, pero puede variar de 8 a 45 años; generalmente comienza con dolor crónico y rigidez de espalda, que es gradual y de comienzo insidioso. Puede llevar un período largo, de promedio al rededor de 6 años, antes de que se diagnostique correctamente.

El patrón habitual de la enfermedad es de una progresión lenta y constante y como tal las complicaciones de la EA tales como fracturas vertebrales, no son infrecuentes, manifestándo se hasta mucho más tarde en la vida.
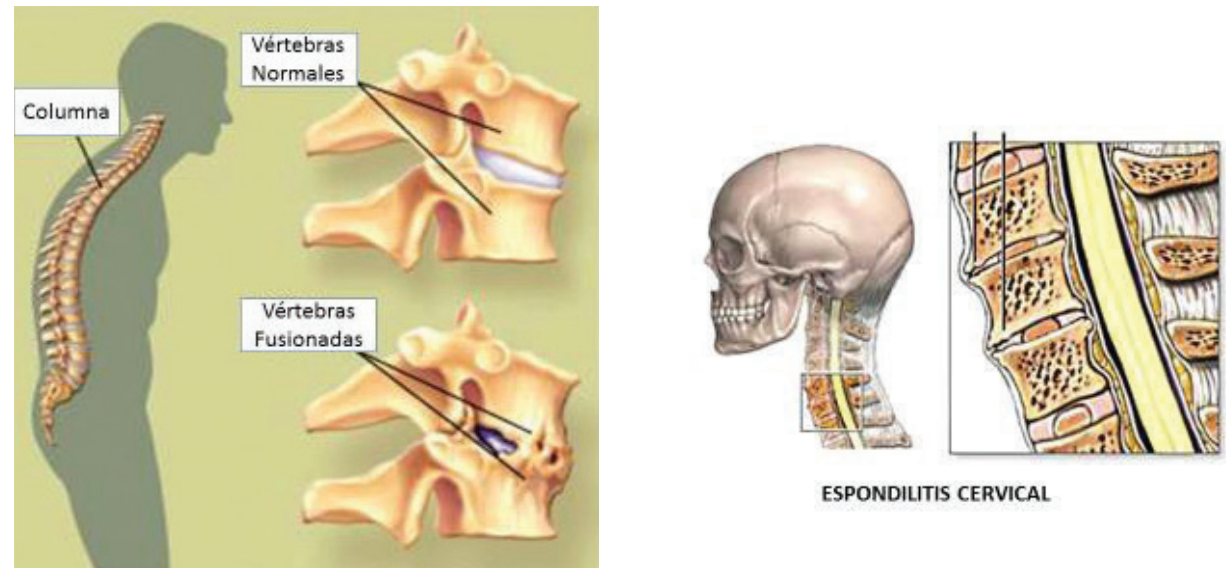

ESPONDILITIS CERVICAL

Fig. 5 - Espondilitis Anquilosante

La incidencia de EA es entre el 0,5 y el 14 por cien mil personas/ año, mientras que la prevalencia de la EA (espondilitis anquilopoyética) asociada a la presencia de antígeno HLAB27 y a sacroileítis oscila en torno al 0,1\% en la población general (Hanson, J.A., Mirza,S.: Predisposition for spinal fracture in ankylosing spondylitis. AJR2000;174:150), el 0,3\% (Dean LE, Jones GT, MacDonald AG, Downham C, Sturrock RD, Macfarlane GJ: Global prevalence of ankylosing spondylitis. Rheumatology (Oxford) 53:650-657,2014), y hasta el 1,4\%; los hombres se afectan aproximadamente el doble que las mujeres (Varones (Einsiedel T, Schmelz A, Arand M, Wilke HJ, Gebhard F, Hartwig E, et al.: Injuries 
of the cervical spine in patients with ankylosing spondylitis : experience at two traumacenters. J Neurosurg Spine 5 : 33-45, 2006, Feldtkeller E, Vosse D, Geusens P, van der Linden S: Prevalence and annual incidence of vertebral fractures in patients with ankylosing spondylitis. Rheumatol Int $26: 234-239,2006$ )

Las espondiloartropatías sero-negativas tienen una predisposición genética relacionada con el complejo mayor de histocompatibilidad clase 1 molécula HLA-B27. Del 90 al 95\% de pacientes con EA son positivos para el HLA-B27, pero la EA se desarrolla en sólo el 5\% de los individuos positivos HLA-B27. Este hallazgo sugiere que otros factores no identificados genéticos y ambientales también juegan un papel significativo en la patogénesis de la EA.

El diagnóstico de la EA se la hace en la base de factores clínicos y radiológicos, conocidos como los criterios modificados de Nueva York (van der Linden S, Valkenburg HA, Cats A. Evaluation of diagnostic criteria for ankylosing spondylitis. A proposal for modification of the New York criteria. Arthritis Rheum 1984; 27: 361-368).

\section{ESPONDILITIS ANQUILOSANTE CRITERIOS DE NEW YORK MODIFICADOS}

Dolor en espalda baja de al menos 3 meses de duración; mejora con ejercicio y no con reposo Limitación del movimiento de la columna lumbar en los planos frontal y sagital

Expansión torácica reducida en relación a los valores normales para edad y sexo. Sacroileítis unilateral grado 3-4

Sacroileítis bilateral grado 2-4

EA definitiva si (41 o 4b) y cualquier criterio clínico (1.3)

Como elemento central en el diagnóstico es la presencia de sacroileitis, definida en las imágenes radiológicas o de resonancia magnética. El grado de sacroileitis se valora de 0 a 4, con el grado correspondiente a articulaciones radiológicamente normales, cambios sospechosos, cambios leves, cambios moderados y anquilosis respectivamente. El dolor inflamatorio de espalda es el hecho clínico central de la EA. La enfermedad puede tener así diferentes estadíos evolutivos, hasta llegar a la calcificación ligamentosa, produciendo una rigidez articular muy intensa. 

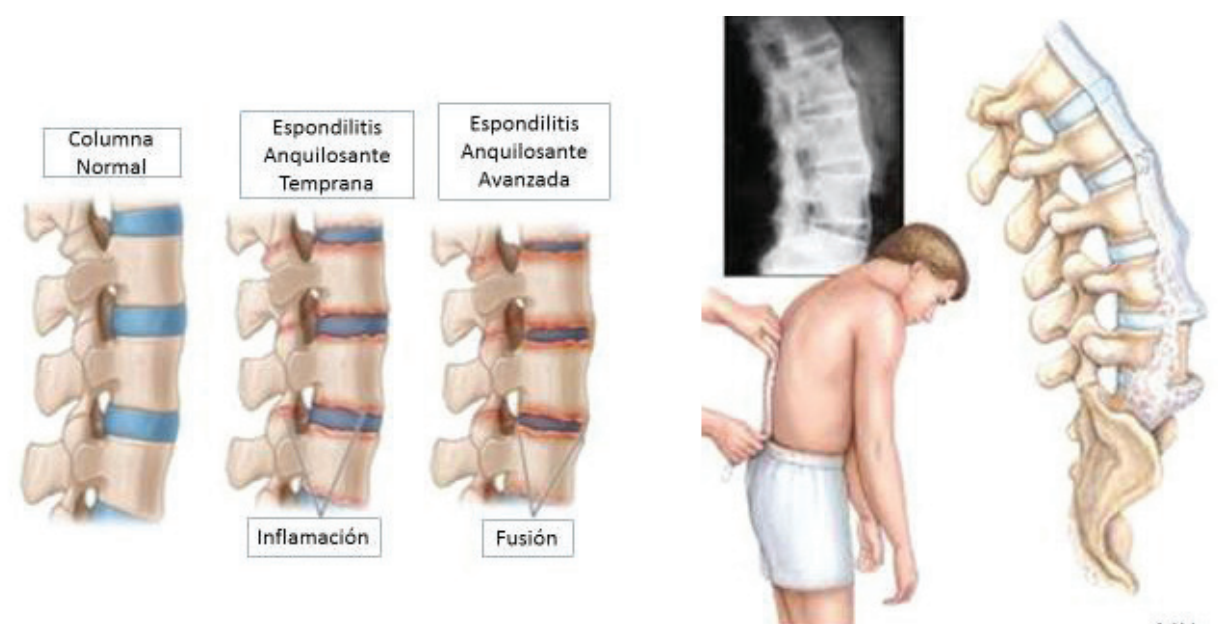

Fig. 6 - Espondilitis Anquilosante - Criterios de New York modificados

El hallazgo de 2 de 4 criterios, sugiere un diagnóstico de dolor inflamatorio de espalda: 1.- rigidez matinal que dura más de 30 minutos, 2.- mejoría del dolor de espalda con la actividad y no con el reposo, 3.- despertar debido a dolor de espalda durante la segunda mitad de la noche, y 4.- dolor alternante en nalgas.

Además de la inflamación del esqueleto axial, puede haber artritis periférica, entesitis y uveítis anterior en la EA; otras manifestaciones en otros órganos, aparatos y sistemas como el pulmonar, cardiovascular o renal son raros pero pueden presentarse.

Respecto a las fracturas de columna vertebral en pacientes con EA, ya que su columna se fusiona a través de osificación ligamentosa y sindemositosis, desarrollan una deformidad como una hipercifosis rígida; a otros niveles, ej. toracolumbar, la rigidez modifica la curvatura lumbar normal - lordosis -, biomecánicamente la columna fusionada es más parecida a un hueso largo y actúa como una palanca rígida, que es incapaz de disipar de forma adecuada la energía de un evento traumático. Esta biomecánica vertebral alterada, combinada a una calidad más débil del hueso osteoporótico del paciente con EA, aumenta importantemente la susceptibilidad a fracturas de columna vertebral, incluso tras traumatismo menores, y a menudo triviales. La columna se conoce como "en caña de bambú". 


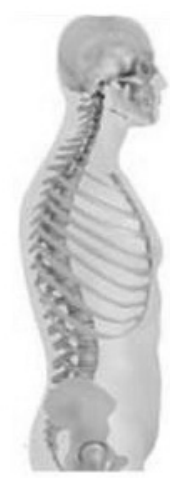

Fig. 7 - Columna sin Espondilitis Anquilosante

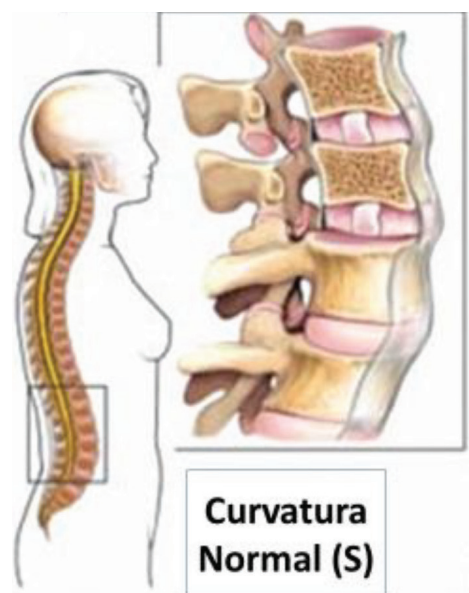

Fig. 9 - Anatomia normal

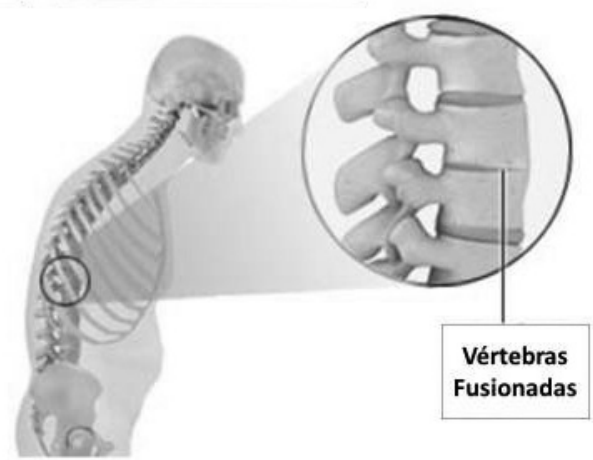

Fig. 8 - Columna con Espondilitis Anquilosante

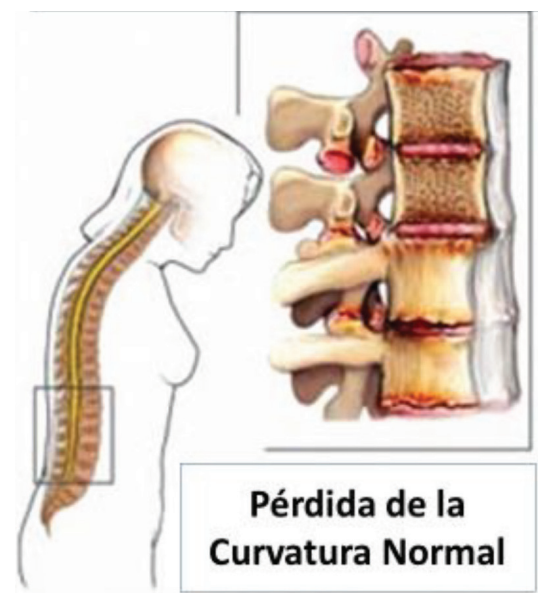

Fig. 10 - Espondilitis Anquilosante

Para aumentar además la susceptibilidad a fracturas vertebrales, la mayoría de pacientes con EA tienen una movilidad significativamente alterada, relacionada directamente a su deformidad de una columna vertebral cifótica y rígida, así como un grado variable de afectación articular periférica; estos factores exacerban la inestabilidad de la marcha y por lo tanto aumentan la susceptibilidad a las caídas que constituye la causa más frecuente de fracturas en estos pacientes. 

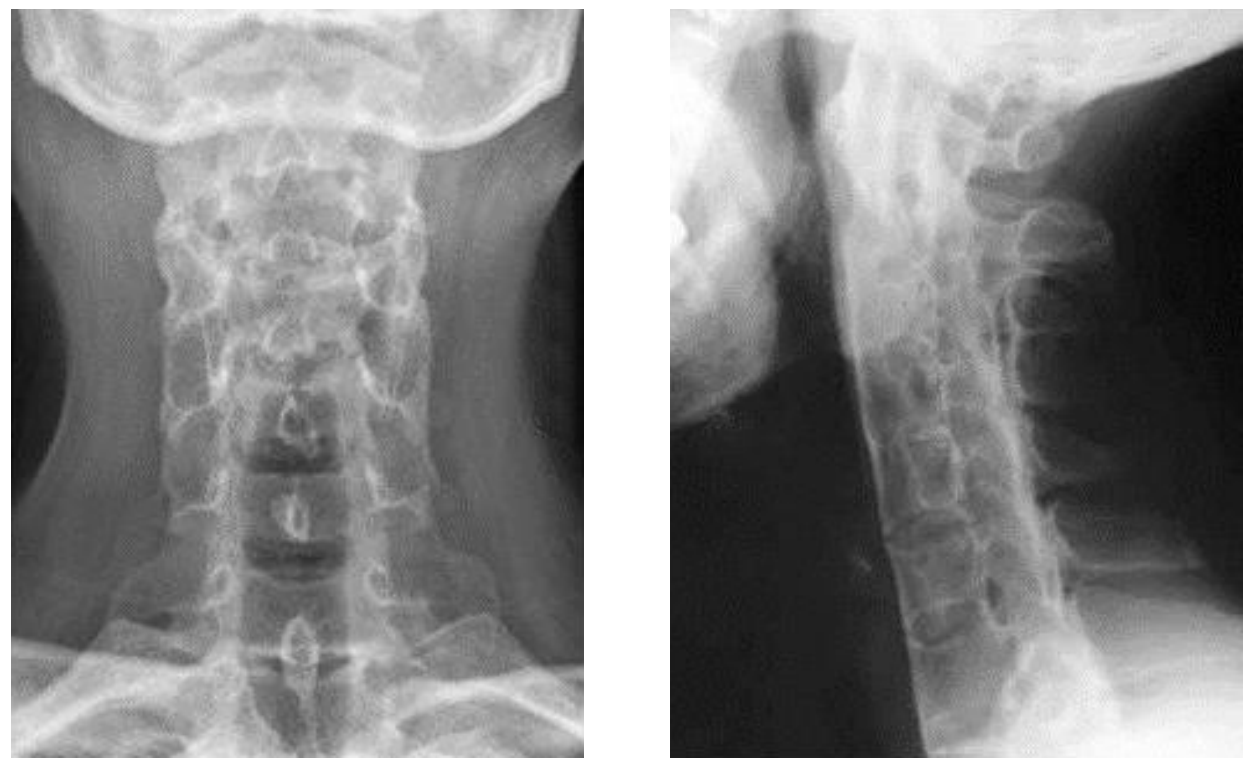

NOTA: NO corresponden al lesionado que comentamos

Fig. 11 - ESPONDILITIS ANQUILOSANTE (formas avanzadas)

Entre los factores de riesgo asociados a fractura vertebral en pacientes con EA, está el sexo (varones), edad ,un índice de masa corporal bajo, osteoporosis, duración de la enfermedad y grado de la formación de sindesmofitos, afectación articular periférica, restricción aumentada del movimiento vertebral y una distancia aumentada del occipital a la pared.

La columna vertebral anquilosada es muy susceptible a las fracturas (Hitchon, P.W., From, A.M., Brenton, M.D., Glaser, J.A.,Torner, J.C.: Fractures of the thora columbar spine complicating ankylosing spondylitis. J Neurosurg Spine 2002; 97: 218-222, Alaranta H, Luoto S, Konttinen YT: Traumatic spinal cord injury as a complication to ankylosing spondylitis. An extended report. Clin Exp Rheumatol 20:66-68, 2002, Cooper C, Carbone L, Michet CJ, Atkinson EJ, O'Fallon WM, Melton LJ III: Fracture risk in patients with ankylosing spondylitis: a population based study. JRheumatol 21:1877-1882, 1994, Olerud C, Frost A, Bring J: Spinal fractures in patients with ankylosing spondylitis. Eur Spine J 5:51-55, 1996, Rowed DW: Management of cervical spinal cord injury in ankylosing spondylitis: the intervertebral disc as a cause of cord compression. J Neurosurg 77:241-246, 1992, Hanson, J.A., Mirza, S.: Predisposition for spinal fracture in ankylosing spondylitis. AJR 

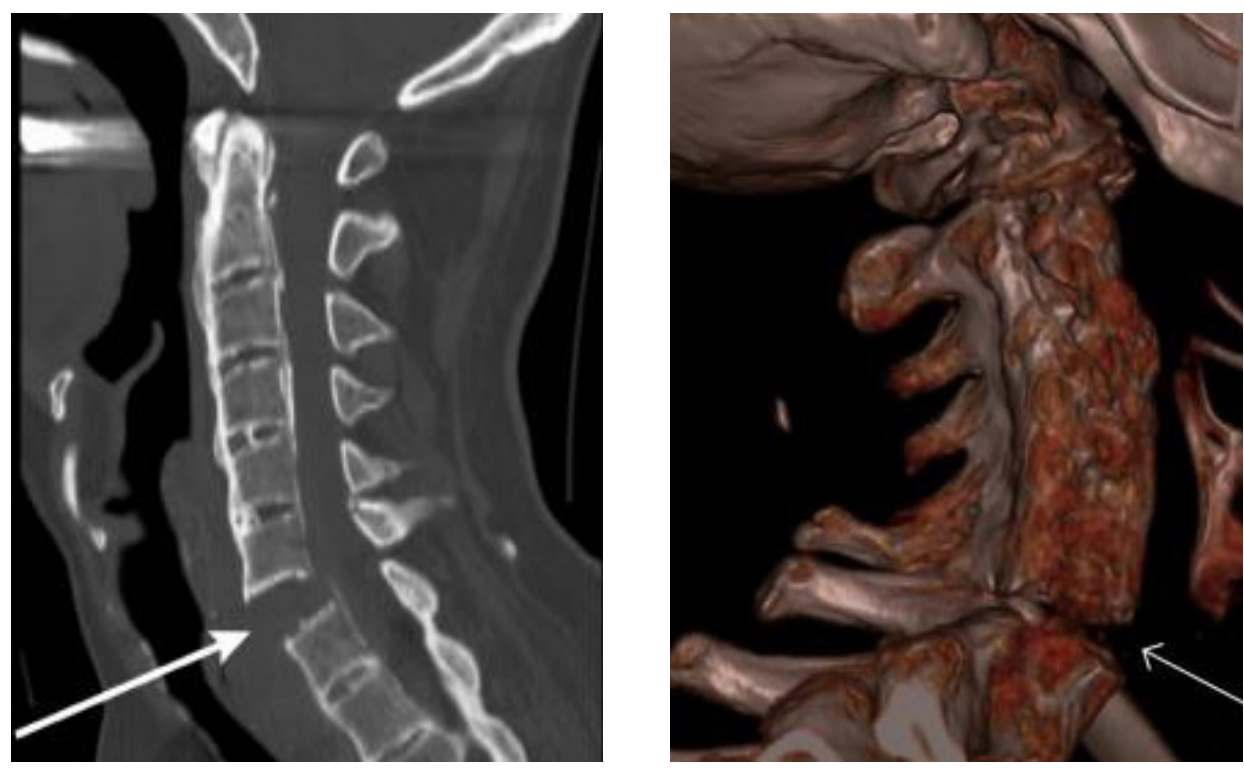

NOTA: NO corresponden al lesionado que comentamos

Fig. 12 - Fracturas cervicales en ESPONDILITIS ANQUILOSANTE

2000; 174: 150.), por la progresiva pérdida de movilidad y osteoporosis secundaria. Sin embargo, aparentemente no se ha logrado establecer una correlación definitiva entre densidad mineral ósea y fracturas vertebrales en estos pacientes (Levine, D.S., Forbat, S.M., Saifuddin, A.: MRI of the axial skeletal manifestations of ankylosing spondylitis. Clin Radiol 2004; 59:400-413)

La incidencia de fractura vertebral en pacientes con EA es entre cuatro veces mayor que en la población general (Hanson, J.A., Mirza, S.: Predisposition for spinal fracture in ankylosing spondylitis. AJR 2000; 174: 150), y hasta 7 veces a nivel toracolumbar, según otros autores (Cooper $C$, CarboneL, Michet CJ, Atkinson EJ, O'Fallon WM, Fracture risk in patients with ankylosing spondylitis: a population based study. J. Reumatol. 1994; 21:1877-82)

Debido a los segmentos anquilosados de la columna vertebral, las fracturas producidas tienen frecuentemente un trazo transversal característicamente y se las ha denominado "fracturas en tallo de zanahoria", 
'carrot stick fractures', (Seong-Bae An, Keung-Nyun Kim, Dong-Kyu Chin, et al., Surgical Outcomes after Traumatic Vertebral Fractures in Patients with Ankylosing Spondylitis, J Korean Neurosurg Soc 56 (2): 108-113,2014).

Las fracturas suelen ser provocadas por un traumatismo menor, como caídas (Bennett, L., Ohashi, K., El-Khoury, G.: Spondyloarhropatles: ankylosing spondylitis and psoriasic arthritis. Radiol Clin N Am, 2004; 42: 121-134), pudiendo pasar desapercibidas inicialmente (Ozgocmen, S., Ardicoglu, O.: Odontoid fracture complicating ankylosing spondylitis. Spinal Cord 2000; 38: 117-119), Finkelstein, J.A., Chapman, J.R., Mirza, S.: Occult vertebral fractures in ankylosing spondylitis. Spinal Cord 1999; 37: 444-447), estando de pie o caminando (el mecanismo más común) o por accidente de tráfico (el segundo más frecuente), -como habría sido en este caso-.

El 75\% de las fracturas ocurre a nivel de la columna cervical, especialmente en la unión cervicotorácica C5-T1 (Bennett, L., Ohashi, K., El-Khoury, G.: Spondyloarhropatles: ankylosing spondylitis and psoriasic arthritis. Radiol Clin N Am, 2004; 42: 121-134, Mitra, D., Elvins, D.M., Speden, D.J., Collins, A.J.: The prevalence of vertebral fractures in mild ankylosing spondylitis and their relationship to bone mineral density. Rheumatol 2000; 39:85-89, Detwiler, K.N., Loftus, C.M., Godersky, J.C., Menezes, A.H.: Management of cervical spine injuries in patients with ankylosing spondylitis. JNeurosurg 1990; 72: 210-215. Exner, G., Bötel, U., Kluger, P., Richter, M., Eggers, C., Ruidisch, M.: Treatment of fracture and complication of cervical spine with ankylosing spondylitis. Spinal Cord 1998; 36: 377-379); en su mayoría, son traumatismos mínimos (Hanson, J.A., Mirza, S.: Predisposition for spinal fracture in ankylosing spondylitis. AJR 2000; 74:150).

Así mismo, la gravedad de la fractura se refleja en las secuelas neurológicas; la incidencia de déficit neurológico oscila entre 40 y $70 \%$, y la tasa de mortalidad es del 35\%, según varios autores, (Hitchon, P.W., From, A.M., Brenton, M.D., Glaser, J.A., Torner, J.C.: Fractures of the thoracolumbar spine complicating ankylosing spondylitis. J NeurosurgSpine 2002;97: 218-222, Taggard, D.A., Traynelis, V.C.: Management of cervical spinal fractures in ankylosing spondylitis with posterior fixation. Spine 25; 16: 2035-2039, Ticó, N., Ramón, S., García-Ortun, et al: Traumatic spinalcord injury complicating ankylosing spondylitis. Spinal Cord 1998; 36: 349-352, Yilmazlar, S., Kocaeli, H., Doygun, M.: Chance type cervical fracture and neurological deficits in ankylosing spondylitis. Ulus Trauma Derg. 2003; 9:76-78.

Por ello es necesario efectuare neste tipo de pacientes con traumatismo espinal un estudio radiológico de todo el neuroeje, y no sólo de la zona sintomática. 
La mayor incidencia de déficits neurológicos secundarios en pacientes con fracturas vertebrales, inicialmente inadvertidas, es de gran importancia en pacientes con EA (Fox, M.W., Onofrio, B.M., Kilgore, J.E.: Neurological complication of ankylosing spondylitis: the intervertebral disc as a cause of cord compression. J Neurosurg 1992; 77: 241- 246, Kauppi, M., Belt, E.A., Soini,l.: "Bamboospine" starts to bend - something is wrong. ClinExpRheumatol 2000; 18: 513-514).

En varias series, se produjo fractura vertebral cervical con una frecuencia del 75\%, (Seong-Bae An, Keung-Nyun Kim, Dong-Kyu Chin, et al., Surgical Outcomes after Traumatic Vertebral Fractures in Patients with Ankylosing Spondylitis, J KoreanNeurosurg Soc 56 (2): 108-113, 2014, Alaranta H, Luoto S, Konttinen YT: Traumatic spinal cord injury as a complication to ankylosing spondylitis. An extended report. Clin Exp Rheumatol 20: 66-68, 2002, Braun J, Sieper J: Ankylosing spondylitis. Lancet 369: 1379-1390, 2007, Foo D, Sarkarati M, Marcelino V: Cervical spinal cord injury complicating ankylosing spondylitis. Paraplegia 23: 358-363, 1985, Lazaro BC, Deniz FE, Brasiliense LB, Reyes PM, Sawa AG, Theodore N, etal: Biomechanics of thoracic short versus long fixation after 3-column injury. JNeurosurg Spine 14: 226-234, 2011, Westerveld LA, van Bemmel JC, Dhert WJ, Oner FC, Verlaan JJ: Clinical outcome after traumatic spinal fractures in patients with ankylosing spinal disorders compared with control patients. Spine J 14: 729-740, 2014).

La parte inferior de la columna vertebral cervical - como en el paciente que comentamos-, se afecta mas frecuentemente, debido a su mayor carga física; la unión toracolumbar es el segundo nivel mas frecuente de lesión en estos pacientes. Las lesiones por hiperextensión, como se ha comentado, son la causa mas frecuente de fractura, aun que también se producen lesiones por hiperflexión, Caron T, Bransford R, Nguyen Q, Agel J, Chapman J, Bellabarba C: Spine fractures in patients with ankylosing spinal disorders. Spine (Phila Pa 1976), Goldberg AL, Keaton NL, Rothfus WE, Daffner RH: Ankylosing spondylitis complicated by trauma: MR findings correlated with plain radiographs and CT. SkeletalRadiol 22: 333-336, 1993, Harding JR, McCall IW, Park WM, Jones BF: Fracture of the cervical spine in ankylosing spondylitis. Br J Radiol 58: 3-7, 1985, Jacobs WB, Fehlings MG: Ankylosing spondylitis and spinal cord injury: origin, incidence, management, and avoidance. Neurosurg Focus 24: E12, 2008, Weinstein PR, Karpman RR, Gall EP, Pitt M: Spinal cord injury, spinal fracture, and spinal stenosis in ankylosing spondylitis. J Neurosurg 57: 609-616, 1982). 

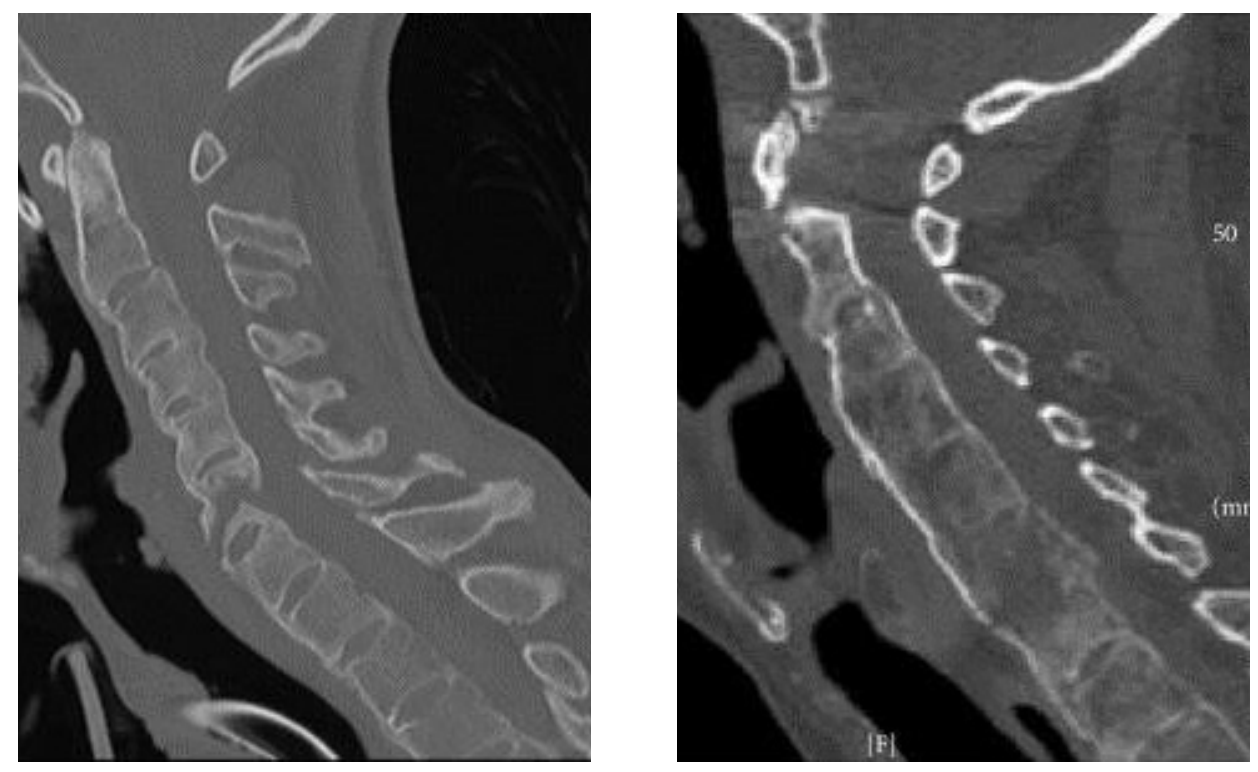

NOTA: NO corresponden al lesionado que comentamos

Fig. 13 - Fracturas cervicales en ESPONDILITIS ANQUILOSANTE

El mecanismo lesional más frecuente en este tipo de lesiones es la hiperextensión-distracción - estadío 2 de Allen-, (A. Carrillo-Colmenero; T. García-de la Oliva y H.F. Cabrera Ortiz, Fractura transdiscal y subluxación C5-C6 en paciente con espondilitis anquilopoyética, Neurocirugía 2006; 17: 440-444, Foo D, Sarkarati M, Marcelino V: Cervical spinalcord injury complicating ankylosing spondylitis. Paraplegia 23: 358-363, 1985 Garza-Mercado R: Traumatic extradural hematoma of the cervical spine. Neurosurgery 24:410-414, 1989, Olerud C, Frost A, Bring J: Spinal fractures in patients with ankylosing spondylitis. Eur Spine J 5: 51-55, 1996,Ticó N, Ramon S, Garcia-Ortun F, Ramirez L, Castelló T, Garcia-Fernández L, et al: Traumatic spinal cord injury complicating ankylosing spondylitis. Spinal Cord 36:349-352, 1998, Caron T, Bransford R, Nguyen Q, Agel J, Chapman J, Bellabarba C: Spine fractures in patients with ankylosing spinal disorders. Spine (Phila Pa 1976), Goldberg AL, Keaton NL, Rothfus WE, Daffner RH: Ankylosing spondylitis complicated by trauma: MR findings correlated with plain radiographs and CT. Skeletal Radiol 22: 333-336, 1993, Harding JR, McCall IW, Park WM, Jones BF: Fracture of the cervical spine in ankylosing spondylitis. BrJRadiol 58:3-7, 1985, Jacobs WB, Fehlings MG: Ankylosing 


\begin{tabular}{|l|l|}
\hline \multicolumn{2}{|c|}{$\begin{array}{c}\text { Clasificación de Allen y Ferguson Classification } \\
\text { (de las lesiones de columma subaxial) }\end{array}$} \\
\hline 1. Flexión-compresión & \\
\hline 2. Compresión Vertical & \\
\hline 3. Flexión-distracción & Estadío 1: Subluxación de carillas \\
\hline & Estadio 2: Luxación Unilateral de carillas \\
\cline { 2 - 3 } & $\begin{array}{l}\text { Estadío 3: Luxación Bilateral de carillas } \\
\text { con 50\% de desplazamiento }\end{array}$ \\
\cline { 2 - 3 } & $\begin{array}{l}\text { Estadío 4: Luxación Completa } \\
\text { (100\% de desplazamiento) }\end{array}$ \\
\hline 4. Extensión-compresión & \\
\hline 5. Extensión-distracción & \\
\hline 6. Flexión Lateral & \\
\hline
\end{tabular}

Quadro 1 - Clasificación de Allen y Ferguson

spondylitis and spinal cord injury: origin, incidence, management, and avoidance. Neurosurg Focus 24: E12, 2008, Weinstein PR, Karpman RR, Gall EP, Pitt M: Spinal cord injury, spinal fracture, and spinal stenosis in ankylosing spondylitis. J Neurosurg 57: 609-616, 1982), produciendo fracturas transdiscales.

El mecanismo flexor suele provocar fracturas a través del cuerpo vertebral, - tipo Chance -, (Yilmazlar, S., Kocaeli, H., Doygun, M.: Chance type cervical fracture and neurological deficits in ankylosing spondylitis. Ulus Trauma Derg. 2003; 9:76-78),

Respecto al pronóstico, la mayor incidencia de lesión medular espinal se relaciona con la mayor incidencia de fracturas vertebrales, fracturas muy inestables, como las de un hueso largo y hematoma epidural tras fractura en pacientes con EA; por lo tanto, el estado neurológico de los pacientes con EA se afecta mas posteriormente por mas procesos complicados, (Westerveld LA, van Bemmel JC, Dhert WJ, Oner FC, Verlaan JJ: Clinical outcome after traumatic spinal fractures in patients with ankylosing spinal disorders compared with control patients. Spine J 14 : 729-740, 2014). 

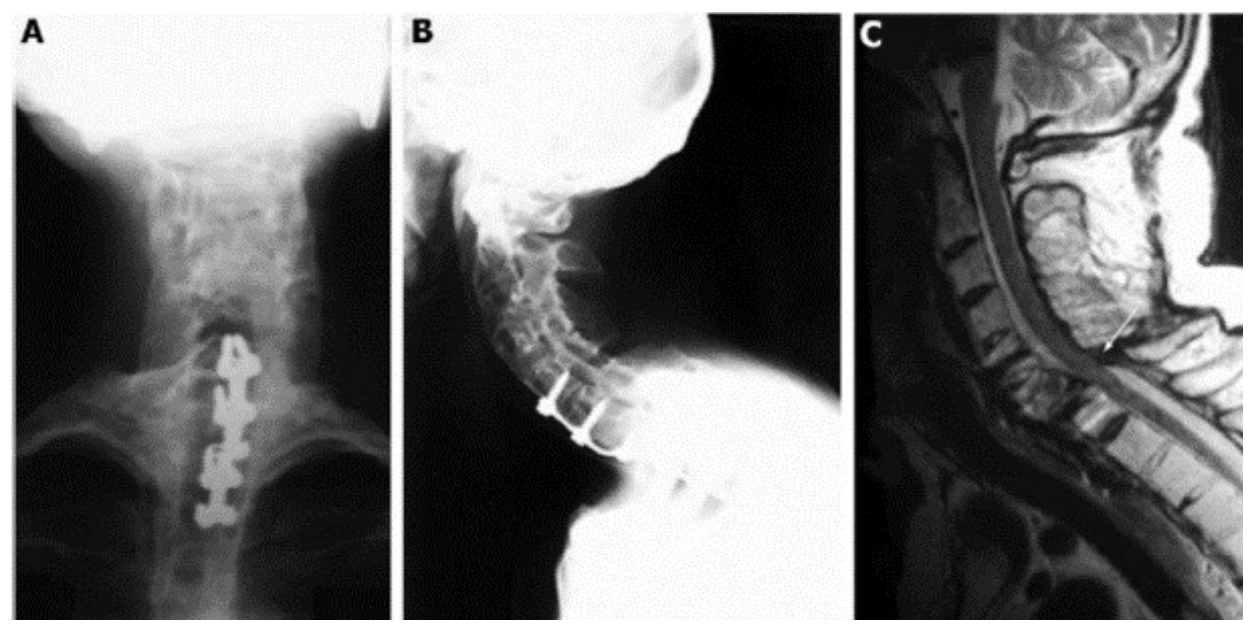

NOTA: NO corresponden al lesionado que comentamos

Fig. 14 - Fracturas cervicales en ESPONDILITIS ANQUILOSANTE

En el accidente que comentamos, consistente en una colisión por alcance posterior, que, en base a las circunstancias y características del accidente, sería una colisión de las denominadas "a baja velocidad", el conductor del turismo Seat Ibiza alcanzado, habría sufrido un mecanismo de los denominados "por latigazo cervical", con unas fuerzas actuantes sobre el cuello, con componentes de extensión y flexión, asociados a rotación e inclinación lateral, y con la producción de unas lesiones que según la clasificación de Quebec de los conocidos como WAD o Whiplash Associated Disorders" - "Trastornos Asociados al Latigazo Cervical", serían de un grado IV - fractura.

En este accidente se ha dado una característica singular, y es que sus consecuencias han sido, aparentemente, desproporcionadamente más graves de lo que habría sido de esperar, en base al tipo y características del accidente.

Esa mayor gravedad de las lesiones se debió a que la fuerza actuante sobre el cuello del conductor lesionado, encontró un cuello patológico, debido a la enfermedad preexistente del conductor, consistente en una Espondilitis Anquilosante, que al volver el cuello mas rígido, así como menos resistente por la osteoporosis asociada que previsiblemente padecía, y que se asocia a la enfermedad, produjeron que un descenso en el umbral lesivo, enfrentado a la misma intensidad de fuerza, causara lesiones que no 


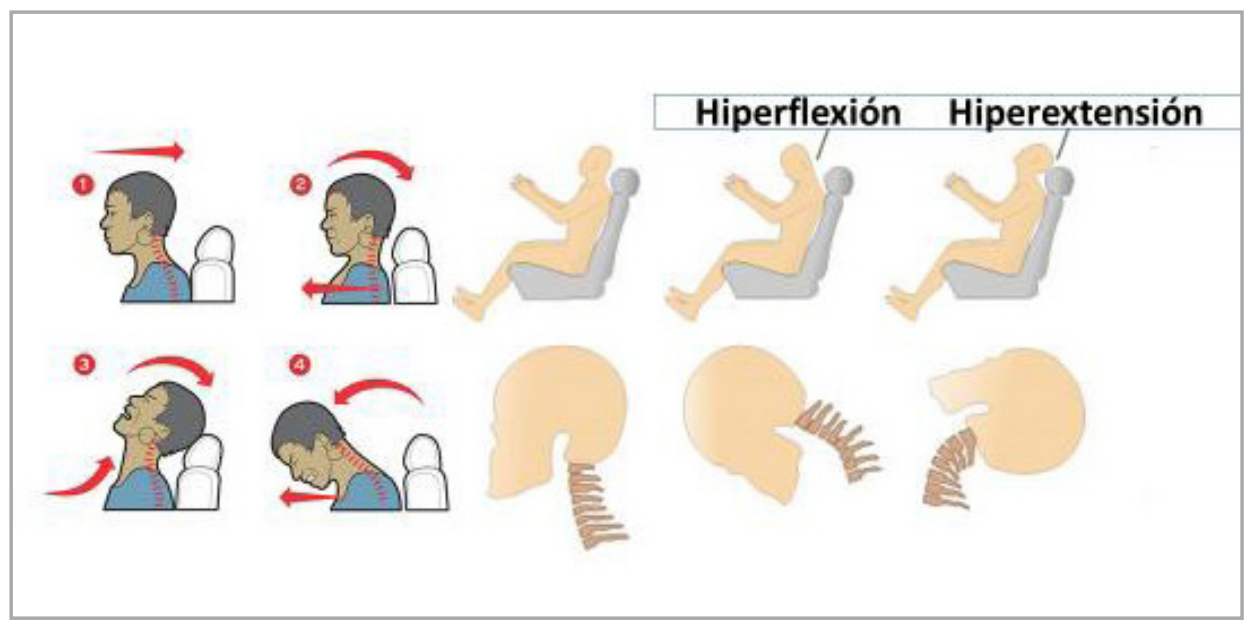

Fig. 15 - Latigazo Cervical - Mecanismo Lesivo

\begin{tabular}{|l|l|}
\hline \multicolumn{2}{|c|}{ Lesiones Asociadas al Latigazo Cervical } \\
\hline GRADO & PRESENTACION CLINICA \\
\hline $\mathbf{0}$ & $\begin{array}{l}\text { Sin molestias en cuello } \\
\text { Sin signos clínicos }\end{array}$ \\
\hline I & $\begin{array}{l}\text { Dolor, rigidez o molestias en cuelo } \\
\text { Sin signos clinicos }\end{array}$ \\
\hline II & $\begin{array}{l}\text { Molestias en cuello y } \\
\text { signos músuloesqueléticos }\end{array}$ \\
\hline III & $\begin{array}{l}\text { Molestias en cuello y } \\
\text { signos neurológicos }\end{array}$ \\
\hline IV & $\begin{array}{l}\text { Molestias en cuello y } \\
\text { fratura o luxación }\end{array}$ \\
\hline
\end{tabular}

Quadro 2 - Lesiones Asociadas al Latigazo Cervical

se habrían producido en un cuello normal para la edad del paciente, sin la patología que éste sufría.

Así, a diferencia de la elasticidad de los ligamentos, discos intervertebrales, etc., de una columna vertebral sin esa patología, la fuerza del movimiento de la cabeza del conductor,con transmisión de fuerza al cuello, 
habría tenido uno componente inicial de extensión, seguido posteriormente de un componente de flexión de la cabeza sobre elcuello, que no habrían producido en este lesionado un desplazamiento de la cabeza con los arcos normales, esperables en otra persona sin patología, sino que esas fuerzas se habrían aplicado sobre unas estructuras rígidas, calcificadas, con menor flexibilidad, produciendo las fracturas citadas.

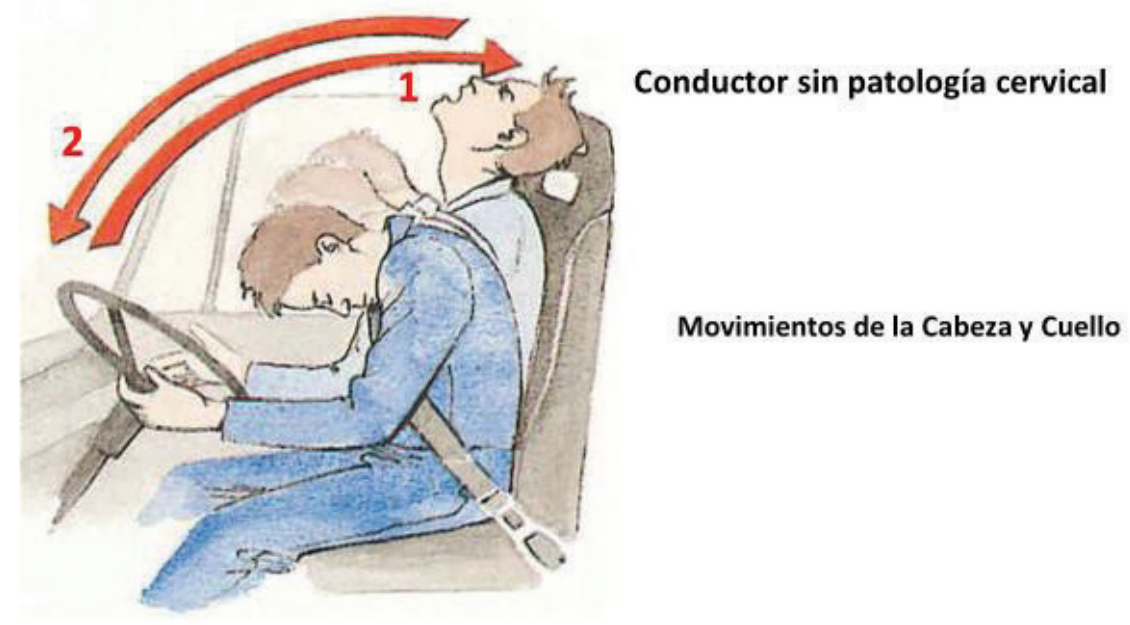

Fig. 16 - Colisión por alcance posterior

En síntesis, se recuerda que la misma intensidad de fuerza derivada de un accidente, por ejemplo como en este caso, consistente en una colisión de las denominadas "alcance posterior a baja velocidad", puede originar diferentes tipos y grados de lesión, en función de la salud o patología previa (el denominado "estado anterior"), del individuo sobre el que actúe esa fuerza, lo cual tiene una gran trascendencia, tanto desde el punto de vista asistencial, por el alto indice de sospecha que debe regir las actuaciones médicas, como desde el puntos de vista pericial o médico-legal, para tratar de establecer o descartar la existencia de nexo causal entre el accidente y la lesión. 